\title{
LIXIVIACIÓN DE PLATA A TEMPERATURAS ALTAS EN MINERAL COMPLEJO DE PIRITA
}

\author{
Américo Suazo Ramos Hurtado* \\ RESUMEN
}

El estudio de investigación se llevó a cabo de una muestra de relave pirita-plata que facilitó la Empresa Administradora Cerro S.A.C. ubicada en el distrito Simón Bolívar, provincia y región de Pasco. La importancia de los aportes de investigación para su mejor tratamiento a este material obedece a que tiene como stock un millón de toneladas con un promedio de ley de $5,14 \mathrm{ozAg} / \mathrm{t}$ y otros dos millones con ley entre 5 a $6 \mathrm{ozAg} / \mathrm{t}$. Estas últimas no han tenido ningún tratamiento previo, por lo tanto, se espera determinar el método adecuado para optimizar la recuperación de plata en este mineral complejo de pirita-plata.

La recuperación de plata en este mineral complejo por el método de flotación alcanzó 20 \%, conllevando a definirlo como un mineral refractario a este proceso, por lo tanto, es materia de investigación otros procesos como lo de la lixiviación.

De la evaluación de resultados de las pruebas de lixiviación, haciendo uso de la matriz de un diseño experimental hexagonal, donde se extrapola resultados para determinar el óptimo, se determina que se puede lograr una recuperación de plata de 50,06 \% (variable dependiente) con un consumo de $\mathrm{NaCN}$ de $4,8 \mathrm{~kg} / \mathrm{t}$ (variable independiente) a una temperatura de $40^{\circ} \mathrm{C}$ (variable independiente).

El tratamiento de este mineral es refractario a la flotación, a través de lixiviación se logra recuperar no a niveles de minerales no complejos, sin embargo, los procedimientos con temperaturas altas requiere de más evaluaciones a nivel laboratorio y a nivel piloto.

Palabras clave: caracterización, lixiviación, temperatura, recuperación, plata, diseño

\section{LIXIVIATION OF SILVER AT HIGH TEMPERATURES IN COMPLEX MINERAL}

\begin{abstract}
The research study was conducted on a sample of pyrite-silver tailings provided by the Administrative Company Cerro S.A.C. located in the Simón Bolívar district, province and region of Pasco. The importance of research contributions for its best treatment of this material is due to the fact that it has a stock of one million tons with an average grade of 5,14 $\mathrm{ozAg} / \mathrm{t}$ and another two million with a grade between 5 to $6 \mathrm{ozAg} / \mathrm{t}$. The latter have not had any previous treatment, therefore, it is expected to determine the appropriate method to optimize the recovery of silver in this pyrite-silver complex mineral.
\end{abstract}

Facultad de Ingeniería Química, Universidad Nacional del Centro del Perú, Junín, Perú a_ramos_h@hotmail.com 
The recovery of silver in this complex mineral by the flotation method reached $20 \%$, leading to define it as a refractory mineral to this process, therefore, it is a matter of investigation other processes such as leaching.

From the evaluation of the results of the leaching tests, making use of the matrix of a hexagonal experimental design, where results are extrapolated to determine the optimum, it is determined that a silver recovery of 50,06 \% can be achieved (dependent variable) with a $\mathrm{NaCN}$ consumption of $4,8 \mathrm{~kg} / \mathrm{t}$ (independent variable) at a temperature of $40^{\circ} \mathrm{C}$ (independent variable).

The treatment of this mineral is refractory to flotation, through leaching it is recovered not at levels of non-complex minerals, however, the procedures with high temperatures require more evaluations at the laboratory level and at the pilot level.

Key words: characterization, leaching, temperature, recovery, silver, design

\section{INTRODUCCIÓN}

La muestra en estudio es un relave de mineral pirita-plata almacenado, cuyo tratamiento por el método de flotación en los años 2011 y 2012 alcanzó sólo el $20 \%$ de recuperación de plata de una cabeza de 6,4 oz/t. Se tiene almacenado este tipo de relave aproximadamente 1000000 de toneladas con ley de 5,14 oz/t.

Por la complejidad del mineral y tener un comportamiento refractario a la flotación, se optó por llevar a cabo trabajos de investigación mediante el proceso de lixiviación.

Teniendo como precedente los resultados de investigación para este mineral, tanto lo ejecutado por la misma empresa, por el Dr. Luis E. Calzada y los resultados de las pruebas preliminares de esta investigación, para poder investigar los efectos sobre la recuperación de plata a diferentes condiciones se procedió a aplicar un diseño hexagonal (Castro Ch. J; 2005: 38 ), tomando como las variables independientes la variación del agente lixiviante $(\mathrm{NaCN})$ y la variación de la temperatura.

La rapidez de disolución de la plata se incrementa linealmente con el aumento en la concentración de cianuro hasta que se alcanza un máximo, más allá del cual un incremento en la concentración no proporciona una disolución del oro y de la plata, sino por el contrario tiene un efecto retardante (Combi L; 1958: 29).

Se han propuesto las siguientes fórmulas para la disolución de la Ag en soluciones de cianuro diluidas: 
1) $4 \mathrm{Ag}+8 \mathrm{NaCN}+\mathrm{O}_{2}+2 \mathrm{H}_{2} \mathrm{O} \rightarrow 4 \mathrm{NaAg}(\mathrm{CN})_{2}+4 \mathrm{NaOH}$

A esta ecuación se le conoce como la ecuación de Elsner.

2) $2 \mathrm{Ag}+4 \mathrm{NaCN}+2 \mathrm{H}_{2} \mathrm{O} \rightarrow 2 \mathrm{NaAg}(\mathrm{CN})_{2}+2 \mathrm{NaOH}+\mathrm{H}_{2}$

Esta ecuación fue sugerida por Janin.

3) $2 \mathrm{Ag}+4 \mathrm{NaCN}+2 \mathrm{H}_{2} \mathrm{O}+\mathrm{O}_{2} \rightarrow 2 \mathrm{NaAg}(\mathrm{CN})_{2}+2 \mathrm{NaOH}+\mathrm{H}_{2} \mathrm{O}_{2}$

$\mathrm{El}$ peróxido de hidrógeno se utiliza en la siguiente ecuación:

4) $2 \mathrm{Ag}+4 \mathrm{NaCN}+\mathrm{H}_{2} \mathrm{O}_{2} \rightarrow 2 \mathrm{NaAg}(\mathrm{CN}) 2+2 \mathrm{NaOH}$

Estas dos últimas ecuaciones fueron sugeridas por Boldaender. Sin embargo, la mayoría de las ecuaciones son muy semejantes a las propuestas por Elsner.

La ecuación de Elsner procederá hasta que todo el cianuro se haya consumido o hasta que todo el metal haya pasado a solución.

Una solución acuosa de cianuro alcalino se hidroliza de la siguiente manera:

$$
\mathrm{NaCN}+\mathrm{H}_{2} \mathrm{O} \rightarrow \mathrm{HCN}+\mathrm{NaOH}
$$

El desarrollo de esta ecuación depende de la cantidad del álcali presente en el cianuro que se encuentra libre, si es considerable entonces la descomposición del cianuro se puede considerar despreciable, teniendo un resultado contrario si la cantidad de álcali es insuficiente.

La adición de cal a la pulpa de cianuro es prácticamente universal, no sólo para impedir la pérdida de cianuro por hidrólisis sino también para neutralizar cualquier constituyente ácido del mineral, el cual liberaría ácido cianhídrico.

En presencia de ácido carbónico se descompone las soluciones de cianuro alcalinas de la siguiente manera:

$$
\mathrm{NaCN}+\mathrm{H}_{2} \mathrm{CO}_{3} \rightarrow \mathrm{HCN}+\mathrm{NaHCO}_{3}
$$

La reacción anterior también se puede impedir por el uso de cal o de otros álcalis.

Influencia de la concentración de cianuro: Julian y Smart (1903), citado por Oyarce y Lescano (2015), opinan que los cianuros de sodio, potasio, amonio, calcio, estroncio y bario tienen el mismo poder disolvente por radical cianuro para el oro y la plata. La velocidad de reacción en la disolución de la plata se incrementa linealmente conforme aumenta la concentración de cianuro hasta alcanzar un máximo, más allá del cual un incremento en la concentración no proporciona mayor disolución del oro ni de la plata, sino por el contrario tiene un efecto retardante, tal como indica la tendencia de la figura 1. 

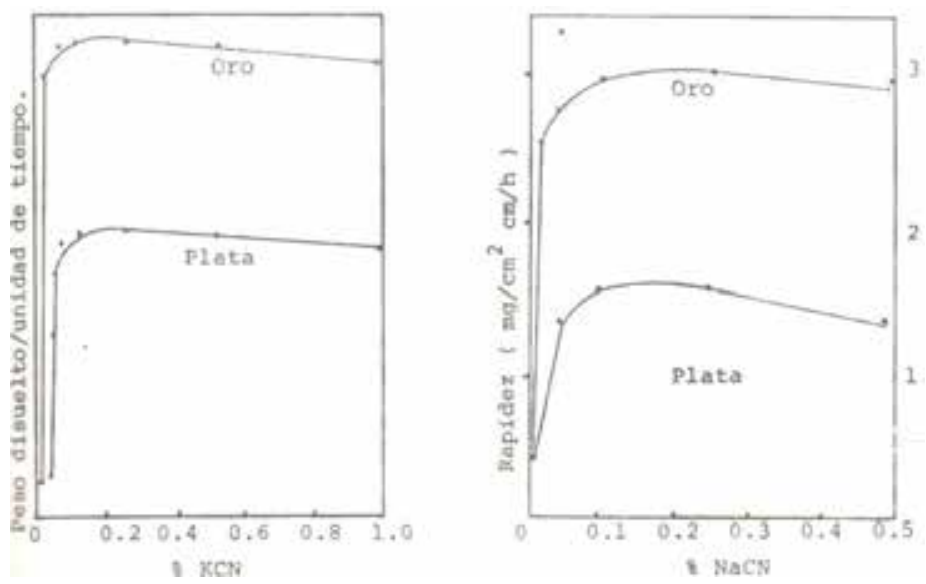

Figura 1. Efecto de la concentración de cianuro sobre la rapidez de disolución del oro y la plata.

La aplicación del calor a una solución de cianuro que contenga plata metálica presenta dos factores opuestos que afectan la rapidez de la disolución. El aumento en la temperatura incrementa la actividad de la solución y por lo tanto, la rapidez de la disolución de los valores metálicos (Julian y Smart 1903 - citado por Oyarce y Lescano - 2015). Al mismo tiempo, la cantidad de oxígeno de la solución disminuye debido a que la solubilidad de los gases decrece con el aumento de la temperatura. El efecto de incrementar la temperatura sobre la rapidez de disolución del oro y la plata se ha investigado por muchos científicos, quienes además pudieron calcular las energías de activación del proceso. La tabla 1 muestra un resumen de los resultados disponibles. La energía de activación de la plata cae en un rango de 2 a $5 \mathrm{kcal} / \mathrm{mol}$.

Tabla 1. Energía de activación para la reacción de disolución de plata en soluciones de cianuro, Julian y Smart (Fuente: Oyarce y Lescano - 2015)

\begin{tabular}{ccccccccc}
\hline & $\begin{array}{c}\text { Temp } \\
{ }^{\circ} \mathrm{C}\end{array}$ & $\begin{array}{c}\mathrm{PO}_{2} \\
(\mathrm{kPa})\end{array}$ & $\begin{array}{c}\text { Conc.\% } \\
\text { KCN }\end{array}$ & $\begin{array}{c}\text { Conc.\% } \\
\mathrm{NaCN}\end{array}$ & $\begin{array}{c}\text { (KOH) } \\
\text { g-mol/1 }\end{array}$ & r.p.m & $\begin{array}{c}\text { Energía de } \\
\text { Activación } \\
(\mathrm{kcal} / \mathrm{mol})\end{array}$ & Investigador \\
\hline $\mathrm{Ag}$ & $15-25$ & 0,21 & 0,043 & 0 & $10^{-1}$ & 0 & 3,20 & Lund \\
$\mathrm{Ag}$ & $24-$ & 3,40 & 0 & 0,18 & 0 & 895 & 2,40 & $\begin{array}{l}\text { Deitz } \\
\text { Halpern y }\end{array}$ \\
& 110 & & & & & & & $\begin{array}{l}\text { Halpen } \\
\text { Kakovskii y } \\
\text { Kholmanskikh }\end{array}$ \\
\hline
\end{tabular}

El uso del oxígeno o de un agente oxidante es esencial para la disolución del oro y la plata bajo condiciones normales de cianuración. Tales agentes oxidantes, como el peróxido de sodio, permanganato de potasio, bromo, cloro, entre otros, se han utilizado con más o menos éxito en épocas anteriores; pero debido al costo elevado que presentan estos reactivos y al manejo de los mismos ya han caído en desuso. Pero como ya se tiene un mayor entendimiento de las reacciones involucradas en el proceso de cianuración y mejor conocimiento de la función que 
desempeñan los constituyentes no deseables en el mineral, han mostrado que una adecuada aereación en condiciones correctas da tan buenos resultados como con el uso de los agentes oxidantes..

Los rendimientos de extracción de plata mediante el proceso de lixiviación ácido-clorurante son sensibles a la granulometría del mineral y la temperatura. Un P80 de $18 \mu \mathrm{m}$ y una temperatura de $60 \mathrm{oC}$ permiten obtener un rendimiento máximo de plata del orden de $55 \%$. Es importante remarcar el menor tiempo $(2 \mathrm{~h})$ de lixiviación para alcanzar este porcentaje (Calzado Luis E: 2012: 4-12).

\section{PARTE EXPERIMENTAL}

Las pruebas de lixiviación se llevaron a cabo en el laboratorio metalúrgico de la Empresa Administradora Cerro S.A.C.

La muestra a lixiviar fue relave pirita-plata con una ley de $5.14 \mathrm{oz} / \mathrm{t}$ y una granulometría de P80 de $54 \mu \mathrm{m}$.

Equipos utilizados:

- $\quad$ Reactor de 2 litros para temperaturas altas

- $\quad$ Dosificadores de reactivos

- Potenciómetro digital

- $\quad$ Estufa universal Memmert

- Balanza analítica

- Tamizadora Ro - tap-

\section{Análisis mineralógico de la muestra}

La caracterización de la muestra fue realizada por un microscopio óptico, equipo de difracción de rayos $\mathrm{X}$ y un microscopio electrónico de barrido (scanning electron microscope, SEM). Los resultados de tales caracterizaciones indican que existen limitantes que impiden una extracción total de la plata, como elemento valioso de la muestra. El principal obstáculo es que la plata se encuentra en un tamaño muy pequeño e incluido dentro de la matriz de la pirita. Esta morfología añadida a la contextura compacta de la pirita (mínimo de porosidad) hace que la exposición del elemento valioso a la solución lixiviante se limite y traiga como consecuencia una disminución a su extracción en la solución impregnada.

El análisis por SEM fue realizado utilizando dos programas de análisis distintos - una específica para fases reducidas (elementos nativos, sulfuros, sulfosales, etc.) y otra específica para fases oxidadas (óxidos, carbonatos, sulfatos y silicatos), de esta manera una serie de granos fueron estudiados cuantitativamente, las que se detallan en las tablas 2 y 3.

Tabla 2. Composición mineralógica aproximada del mineral

\begin{tabular}{lr}
\hline Pirita & $70-75 \%$ peso \\
Cuarzo & $17-19 \%$ peso \\
Hinsdalita & $0,9-1,0 \%$ peso \\
Cobre gris + sulfoantimoniuro de $\mathrm{Cu} / \mathrm{Pb}$ & $0,4-0,6 \%$ peso \\
Galena + sulfato de plomo & $0,6-0,8 \%$ peso \\
Blenda & $0,1-0,3 \%$ peso \\
\hline
\end{tabular}


Tabla 3. Textura mineralógica del mineral compuesto

\begin{tabular}{|c|c|c|c|}
\hline Nombre & Forma & $\begin{array}{l}\text { Abundancia } \\
\text { Relativa } \\
\end{array}$ & $\begin{array}{l}\text { Tamaño de } \\
\text { Grano }(\mu \mathrm{m})\end{array}$ \\
\hline \multirow[t]{7}{*}{ Pirita } & Libre & I & $20-200$ \\
\hline & Asociado al cuarzo & I & 200 \\
\hline & Asociado a la galena & III & 30 \\
\hline & $\begin{array}{l}\text { Presenta inclusiones de } \\
\text { chalcopirita }\end{array}$ & III & $<5$ \\
\hline & $\begin{array}{l}\text { Presenta inclusiones de cobre } \\
\text { gris }\end{array}$ & III & $<5$ \\
\hline & $\begin{array}{l}\text { Presenta inclusiones de sulfosal } \\
\text { rico en bismuto }\end{array}$ & III & $<5$ \\
\hline & Asociada a la blenda & III & $<20$ \\
\hline \multirow{3}{*}{ Cuarzo } & Libre & I & $100-300$ \\
\hline & Asociado a la pirita & I & 200 \\
\hline & Asociado al Al, S, P, Pb, Ba, Ca & II & $15-20$ \\
\hline \multirow[t]{2}{*}{ Galena } & Libre & III & $10-70$ \\
\hline & Asociada a la pirita & III & $<30$ \\
\hline \multirow[t]{2}{*}{ Blenda } & Libre & III & $<80$ \\
\hline & Asociada a la pirita & III & $<20$ \\
\hline \multirow[t]{4}{*}{ Tenantita } & Libre & III & $30-100$ \\
\hline & Inclusiones en la pirita & III & $<5$ \\
\hline & Asociado a la pirita & III & $<5$ \\
\hline & $\begin{array}{l}\text { Presenta enriquecimiento } \\
\text { puntual de plata }\end{array}$ & II & $<3$ \\
\hline \multirow[t]{3}{*}{ Tetrahedrita } & Libre & III & $30-100$ \\
\hline & Inclusiones en la pirita & III & $<5$ \\
\hline & $\begin{array}{l}\text { Presenta enriquecimiento } \\
\text { puntual de plata }\end{array}$ & & $<3$ \\
\hline \multirow[t]{2}{*}{ Proustita } & Libre & III & $<12$ \\
\hline & Mixta con la pirita & III & \\
\hline Hinsdalita & Libre & III & $>300$ \\
\hline Covelita & Libre & III & $<5$ \\
\hline Cuprita & Libre & III & $<5$ \\
\hline Freibergita & Libre & III & $<5$ \\
\hline $\begin{array}{l}\text { Sulfoantimoniuro } \\
\text { de Fe y Ag }\end{array}$ & Libre & III & $<5$ \\
\hline $\begin{array}{l}\text { Sulfosal rico en } \\
\text { Bismuto }\end{array}$ & Inclusiones en la pirita & III & 6 \\
\hline Sulfuro de Plata & Libre & III & $<12$ \\
\hline $\begin{array}{l}\text { Sulfuro de } \mathrm{Ag} \text {, } \\
\mathrm{Cu}, \mathrm{Fe}\end{array}$ & Libre & III & $<12$ \\
\hline $\begin{array}{l}\text { Sulfoarseniuro de } \\
\mathrm{Ag}, \mathrm{Cu}, \mathrm{Fe}\end{array}$ & Libre & III & $<12$ \\
\hline Oxidos de Fierro & Libre & III & \\
\hline
\end{tabular}




\section{Pruebas de lixiviación}

Las pruebas preliminares se llevaron a cabo considerando los parámetros descritos en la tabla 4 y tabla 5, donde la tabla 4 describe el estándar de lixiviación utilizado en la Empresa Administradora Cerro SAC.

Tabla 4. Variables fijas en la lixiviación

\begin{tabular}{lc}
\hline \multicolumn{1}{c}{ VARIABLES FIJAS } & VALOR \\
\hline Peso de relave & $0,50 \mathrm{~kg}$ \\
Peso de solución & $0,61 \mathrm{~kg}$ \\
$\mathrm{P}_{80}$ & $54 \mu \mathrm{m}$ \\
Tiempo de oxidación & 8 horas \\
$\mathrm{pH}$ & 10,8 \\
$\mathrm{H}_{2} \mathrm{O}_{2}$ al $50 \%$ & $9,2 \mathrm{~kg} / \mathrm{TM}$ \\
Porcentaje de sólidos & $45 \%$ \\
Dilución $(\mathrm{L} / \mathrm{S})$ & 1,22 \\
\hline
\end{tabular}

Tabla 5. Variables de estudio en la lixiviación

\begin{tabular}{lc}
\hline \multicolumn{1}{c}{ VARIABLES DE ESTUDIO } & VALOR \\
\hline NaCN & 3600 ppm, 5500 ppm y 7000 ppm \\
Período de cianuración & $12 \mathrm{~h}, 24 \mathrm{~h}, 48 \mathrm{~h} \mathrm{y} 72 \mathrm{~h}$ \\
\hline
\end{tabular}

Los resultados se muestran en la tabla 6 , donde se determina que con una concentración de 5 500 ppm de $\mathrm{NaCN}$ en un período de cianuración de 48 horas, se obtiene la mejor recuperación de plata $(47,2 \%)$.

Tabla 6. Balance de plata

\begin{tabular}{cccccccc}
\hline (NaCN ) ppm & Cab. E. & Cab. C. & Cola & Ext. 12 h & Ext. 24 h & Ext. 48 h & Ext. 72 h \\
& oz/t & oz/t & oz/t & $\%$ & $\%$ & $\%$ & $\%$ \\
\hline 3600 & 5,1 & 5,0 & 2,8 & 40,6 & 41,6 & 44,5 & 43,8 \\
5500 & 5,1 & 5,4 & 2,9 & 43,9 & 45,9 & 47,2 & 44,7 \\
7000 & 5,1 & 5,3 & 2,9 & 41,7 & 43,6 & 45,5 & 43,1 \\
\hline
\end{tabular}

Las pruebas finales se llevaron a cabo a través de un diseño hexagonal, donde los detalles se muestran en las tablas 7, 8 y 9. La tabla 7 muestra el período de cianuración óptimo obtenido en sus experimentos del Dr. L. Calzada, donde sólo se utiliza 2 hr de período de cianuración para obtener la más alta recuperación a temperaturas altas, mientras que en la tabla 8 definimos los rangos de evaluación de las dos variables independientes que influyen con mayor relevancia para obtener la variable dependiente (\% de recuperación de $\mathrm{Ag})$. 
Tabla 7. Variables fijas en la lixiviación

\begin{tabular}{ll}
\hline VARIABLES FIJAS & VALOR \\
\hline Peso de relave & $0,50 \mathrm{~kg}$ \\
Peso de solución & $0,61 \mathrm{~kg}$ \\
$\mathrm{P} 80$ & $54 \mu \mathrm{m}$ \\
Tiempo de oxidación & 8 horas \\
Período cianuración & 2 horas \\
$\mathrm{pH}$ & 10,8 \\
$\mathrm{H}_{2} \mathrm{O}_{2}$ al $50 \%$ : & $9,2 \mathrm{~kg} / \mathrm{t}$ \\
Porcentaje de sólidos & $45 \%$ \\
Dilución $(\mathrm{L} / \mathrm{S})$ & 1,22 \\
\hline
\end{tabular}

Tabla 8. Variables de estudio en la lixiviación

\begin{tabular}{ll}
\hline VARIABLES DE ESTUDIO & VALOR \\
\hline NaCN $\left(\mathrm{X}_{1}\right)$ & $3-6 \mathrm{~kg} / \mathrm{t}$ \\
Temperatura $\left(\mathrm{X}_{2}\right)$ & $20-60^{\circ} \mathrm{C}$ \\
\hline
\end{tabular}

Haciendo uso del diseño hexagonal para pruebas experimentales determinaremos un modelo de segundo orden a fin de procesar e identificar los valores que conllevan a determinar la recuperación óptima de plata en los rangos de evaluación de las variables de estudio de la tabla 8 , considerando sus rangos mínimos y máximos para cada uno de ellos.

Para ello se llevará a cabo nueve pruebas, seis puntos experimentales correspondientes a un hexágono y tres puntos replicados en el centro de un plano cartesiano de -1 a 1 y es descrito apropiadamente mediante un modelo matemático de segundo orden y una superficie de respuesta que represente la región óptima de recuperación.

Tabla 9. Valores de variables independientes para las pruebas a nivel experimental.

\begin{tabular}{ccccc}
\hline & \multicolumn{2}{c}{ NIVEL DE DISEÑ } & \multicolumn{2}{c}{ NIVEL EXPERIMENTAL } \\
& \multicolumn{2}{c}{ HEXAGONAL } & \multicolumn{2}{c}{ NaCN, kg/t Temperatura, ${ }^{\circ} \mathrm{C}$} \\
PRUEBAS & $\mathbf{X}_{\mathbf{1}}$ & $\mathbf{X}_{\mathbf{2}}$ & $\mathbf{X}_{\mathbf{1}}$ & $\mathbf{X}_{\mathbf{2}}$ \\
\hline $\mathbf{1}$ & 1 & 0 & 6 & 40,00 \\
$\mathbf{2}$ & 0,5 & 0,866 & 5,25 & 57,32 \\
$\mathbf{3}$ & $-0,5$ & 0,866 & 3,75 & 57,32 \\
$\mathbf{4}$ & -1 & 0 & 3 & 40,00 \\
$\mathbf{5}$ & $-0,5$ & 0,866 & 3,75 & 22,68 \\
$\mathbf{6}$ & 0,5 & 0,866 & 5,25 & 22,68 \\
$\mathbf{7}$ & 0 & 0 & 4,5 & 40,00 \\
$\mathbf{8}$ & 0 & 0 & 4,5 & 40,00 \\
$\mathbf{9}$ & 0 & 0 & 4,5 & 40,00 \\
\hline
\end{tabular}




\section{Determinación de resultados:}

Correspondiendo para el diseño una fórmula cuadrática paraboloide elíptico, según Castro Ch. J; 2005: 38:

$\mathrm{Y}=\mathrm{B}_{0}+\mathrm{B}_{1} * \mathrm{X}_{1}+\mathrm{B}_{2} * \mathrm{X}_{2}+\mathrm{B}_{11} * \mathrm{X}_{12}+\mathrm{B}_{22} * \mathrm{X}_{22}+\mathrm{B}_{12} * \mathrm{X}_{1}^{*} \mathrm{X}_{2} \ldots \ldots . .($ Ecuación 1$)$

Tabla 10. Constantes de ajuste en la ecuación 1

\begin{tabular}{cl}
\hline CONSTANTES & \multicolumn{1}{c}{ VALOR } \\
$\mathrm{B}_{0}$ & 0,23094688 \\
$\mathrm{~B}_{1}$ & 0,7 \\
$\mathrm{~B}_{2}$ & $-0,05773672$ \\
$\mathrm{~B}_{11}$ & $-1,21666667$ \\
$\mathrm{~B}_{12}$ & 0,23094688 \\
$\mathrm{~B}_{22}$ & $-0,95005574$ \\
\hline
\end{tabular}

Con un nivel de significación de 0,05 el modelo matemático obtenido en el diseño hexagonal es significativo, teniendo como resultado $\mathrm{R}^{2}=0,9845$.

Tabla 11. Respuestas según experimento y modelo matemático de la ecuación 1.

\begin{tabular}{ccc}
\hline PRUEBA & $\begin{array}{c}\text { \% RECUPERACIÓN } \\
\text { POR EXPERIMENTO }\end{array}$ & $\begin{array}{c}\text { \% RECUPERACIÓN } \\
\text { POR ECUACIÓN }\end{array}$ \\
\hline 1 & 49,50 & 49,45 \\
2 & 49,30 & 49,35 \\
3 & 48,50 & 48,45 \\
4 & 48,00 & 48,05 \\
5 & 48,80 & 48,75 \\
6 & 49,20 & 49,25 \\
7 & 50,00 & 49,97 \\
8 & 49,80 & 49,97 \\
9 & 50,10 & 49,97 \\
\hline
\end{tabular}


Tabla 12. Recuperaciones de plata (\%) por modelamiento matemático según ecuación (1).

\begin{tabular}{ccccccccccccc}
\hline $\mathrm{NaCN}$ & ${ }^{\circ} \mathrm{C}$ & 20,00 & 24,00 & 28,00 & 32,00 & 36,00 & 40,00 & 44,00 & 48,00 & 52,00 & 56,00 & 60,00 \\
$\mathrm{Kg} / \mathrm{t}$ & $\mathbf{X}_{\mathbf{1}} \backslash \mathbf{X}_{\mathbf{2}}$ & -1 & $-0,8$ & $-0,6$ & $-0,4$ & $-0,2$ & 0 & 0,2 & 0,4 & 0,6 & 0,8 & 1 \\
\hline 3 & -1 & 47,39 & 47,67 & 47,88 & 48,01 & 48,07 & 48,05 & 47,95 & 47,78 & 47,53 & 47,21 & 46,81 \\
3,3 & $-0,8$ & 47,92 & 48,21 & 48,43 & 48,57 & 48,64 & 48,63 & 48,54 & 48,38 & 48,14 & 47,83 & 47,44 \\
3,6 & $-0,6$ & 48,35 & 48,66 & 48,88 & 49,04 & 49,11 & 49,11 & 49,03 & 48,88 & 48,65 & 48,34 & 47,96 \\
3,9 & $-0,4$ & 48,69 & 49,00 & 49,24 & 49,40 & 49,48 & 49,49 & 49,42 & 49,28 & 49,06 & 48,76 & 48,39 \\
4,2 & $-0,2$ & 48,93 & 49,25 & 49,50 & 49,67 & 49,76 & 49,78 & 49,72 & 49,58 & 49,37 & 49,09 & 48,72 \\
4,5 & 0 & 49,07 & 49,40 & 49,66 & 49,84 & 49,94 & 49,97 & 49,92 & 49,79 & 49,59 & 49,31 & 48,96 \\
4,8 & 0,2 & 49,12 & 49,46 & 49,72 & 49,91 & 50,02 & 50,06 & 50,02 & 49,90 & 49,71 & 49,44 & 49,10 \\
5,1 & 0,4 & 49,07 & 49,42 & 49,69 & 49,89 & 50,01 & 50,05 & 50,02 & 49,91 & 49,73 & 49,47 & 49,14 \\
5,4 & 0,6 & 48,92 & 49,28 & 49,56 & 49,76 & 49,89 & 49,95 & 49,93 & 49,83 & 49,66 & 49,41 & 49,08 \\
5,7 & 0,8 & 48,67 & 49,04 & 49,33 & 49,55 & 49,68 & 49,75 & 49,74 & 49,65 & 49,48 & 49,24 & 48,92 \\
6 & 1 & 48,33 & 48,70 & 49,00 & 49,23 & 49,38 & 49,45 & 49,45 & 49,37 & 49,21 & 48,98 & 48,67 \\
\hline
\end{tabular}

\section{RESULTADOS Y DISCUSIÓN}

De las pruebas preliminares de lixiviación con el mineral en estudio a una granulometría de $54 \mu \mathrm{m}$ (relave del proceso de flotación), se determina que el $\mathrm{NaCN}$ a una concentración de 5500 ppm ofrece la mejor recuperación de plata por el método de lixiviación (47,2 \%), siendo el consumo de $\mathrm{NaCN}$ de 4,77 kg/t (tabla 6). Por lo tanto, consideramos la influencia del consumo de cianuro muy determinante para la recuperación de plata. También considerando la relevancia de la temperatura de sólo 2 horas de lixiviación en los experimentos de L. Calzada, para lograr una optimización a través del diseño de experimentos hexagonal, consideramos al consumo de $\mathrm{NaCN}$ y la temperatura como nuestras variables independientes (tabla 8) y la variable dependiente al \% de recuperación de Ag.

En función a la matriz del diseño hexagonal y los rangos establecidos para los experimentos (tabla 9), se ejecuta la pruebas y los resultados de recuperación se nombra en la tabla 11, donde también figura los resultados obtenidos al calcular haciendo uso de la ecuación matemática (1). Con un nivel de significación de 0,05 el modelo matemático obtenido en el diseño hexagonal es significativo, teniendo como resultado $\mathrm{R}^{2}=0,9845$. Por lo tanto, es un modelo matemático confiable para proyectar resultados.

Construyendo una tabla de resultados haciendo uso de la ecuación (1) determinamos que el óptimo bajo los rangos de estudio de las variables dependientes, se puede obtener una recuperación de $50,06 \%$ de $\mathrm{Ag}$ con un consumo de $\mathrm{NaCN}$ de $4,8 \mathrm{~kg} / \mathrm{t}$ y $40{ }^{\circ} \mathrm{C}$ de temperatura (tabla 12).

Las variables del proceso de lixiviación para lograr una máxima recuperación de plata proyectada se detalla en la tabla 13 . 
Tabla 13. Variables de proceso de lixiviación óptimo.

\begin{tabular}{|c|c|}
\hline VARIABLES & VALOR \\
\hline Peso de relave & $0,50 \mathrm{~kg}$ \\
\hline Peso de solución & $0,61 \mathrm{~kg}$ \\
\hline P80 & $54 \mu \mathrm{m}$ \\
\hline Tiempo & 8 horas \\
\hline Tiempo & 2 horas \\
\hline $\mathrm{pH}$ & 10,8 \\
\hline $\mathrm{H}_{2} \mathrm{O}_{2}$ al $50 \%$ : & $9,2 \mathrm{~kg} / \mathrm{t}$ \\
\hline Porcentaje de & $45 \%$ \\
\hline dilución $(\mathrm{L} / \mathrm{S})$ & 1,22 \\
\hline $\mathrm{NaCN}(5500 \mathrm{ppm})$ & $4,8 \mathrm{~kg} / \mathrm{t}$ \\
\hline Temperatura & $40^{\circ} \mathrm{C}$ \\
\hline
\end{tabular}

\section{CONCLUSIONES}

- Es viable la recuperación de plata de los relaves pirita-plata a través del proceso de lixiviación, logrando alcanzar 50,06 \% de recuperación de plata (tabla 12) haciendo uso como agente lixiviante el cianuro de sodio con un consumo de $\mathrm{NaCN}$ de $4,8 \mathrm{~kg} / \mathrm{t}$ y $40{ }^{\circ} \mathrm{C}$ de temperatura.

- El cianuro de sodio aplicado a este mineral tiene mejor lixiviación a una concentración de 5500 ppm (tabla 6).

\section{AGRADECIMIENTO}

A la Empresa Administradora Cerro S.A.C. por haberme permitido llevar a cabo las pruebas de investigación y al Ing. Marco Coronel Soto, responsable del laboratorio metalúrgico, por la información emitida y el apoyo en las pruebas experimentales. 


\section{REFERENCIAS BIBLIOGRÁFICAS}

1. Cambi L. Cyanidation of gold and analogous metals by hidrocyanic acid and oxygen; Att, Accad, nazl Lincei, Rend. Classes sci. fis. 1958; 24: 20-56.

2. Julian H, Smart E. Cyaniding gold and silver ores. London: Charles Griffin and Company Ltd.; 1904.

3. Calzado LE. Estudio del comportamiento a la lixiviación de la pirita de Cerro de Pasco con contenido de plata. Cerro de Pasco: Empresa Administradora Cerro S.A.C.; 2012.

4. Parga JR, Carrillo FR. Avances en los métodos de recuperación de oro y plata de minerales refractarios. Rev Met Madrid. 1996; 32(4): 254-266.

5. Canseco DE. Metalurgia del oro y plata. Lima: Editorial UNI; 1978, pp 44- 147.

6. Meza L. Aspectos fundamentales de los procesos hidrometalúrgicos de los metales preciosos. Medellín-Colombia; 1981. p. 1-80.

7. Temolada J. Optimización del Proceso de Recuperación de Oro por Cianuración Mediante Variantes Hidrometalúrgicas en el Tratamiento de Concentrados Piritosos Refractarios. II Simposio de Geología, Minas y Metalurgia del Oro; junio-julio 1993; Lima-Perú.

8. Castro ChJ. Optimización del proceso de flotación de concentrado de zinc en la Compañía Minera "Yauliyacu" S.A. mediante diseños experimentales. [Tesis]. Lima: Universidad Nacional Mayor de San marcos; 2005. 